\title{
Modified gateway system for double shRNA expression and Cre/lox based gene expression
}

\author{
Nikolina Radulovich ${ }^{1,2}$, Lisa Leung ${ }^{1,3}$, Ming-Sound Tsao ${ }^{1,2,3^{*}}$
}

\begin{abstract}
Background: The growing need for functional studies of genes has set the stage for the development of versatile tools for genetic manipulations.

Results: Aiming to provide tools for high throughput analysis of gene functions, we have developed a modified short hairpin RNA (shRNA) and gene expression system based on Gateway Technology. The system contains a series of entry and destination vectors that enables easy transfer of shRNA or CDNA into lentiviral expression systems with a variety of selection or marker genes (i.e. puromycin, hygromycin, green fluorescent protein-EGFP, yellow fluorescent protein-YFP and red fluorescent protein-dsRed2). Our shRNA entry vector pENTR.hU6.hH1 containing two tandem human shRNA expression promoters, $\mathrm{H} 1$ and $\mathrm{U} 6$, was capable of co-expressing two shRNA sequences simultaneously. The entry vector for gene overexpression, pENTR.CMV.ON was constructed to contain CMV promoter with a multiple cloning site flanked by loxP sites allowing for subsequent Cre/lox recombination. Both shRNA and CDNA expression vectors also contained attL sites necessary for recombination with attR sites in our destination expression vectors. As proof of principle we demonstrate the functionality and efficiency of this system by testing expression of several cDNA and shRNA sequences in a number of cell lines.

Conclusion: Our system is a valuable addition to already existing library of Gateway based vectors and can be an essential tool for many aspects of gene functional studies.
\end{abstract}

\section{Background}

In the era of whole genome sequencing and proteomics, there has never been a greater need to develop versatile tools for gene functional studies. Such studies necessitate a series of genetic manipulations including overexpression and/or downregulation of genes of interest either in in vivo and/or in vitro settings. Downregulation of genes has been made possible by RNA interference (RNAi) technology [1] and targeting genes using expressed short hairpin RNA [2,3] is currently the method of choice by a majority of researchers. The most successful RNAi libraries based on retroviral [4] and lentiviral expression $[5,6]$ of shRNAs have also been utilized for large-scale functional genomic screens [6].

Many gene expression platforms have been developed over years that allow for the constitutive or inducible expression of genes/shRNAs [2,3]. Furthermore, several

\footnotetext{
*Correspondence: ming.tsao@uhn.on.ca

'University Health Network, Ontario Cancer Institute/Princess Margaret Hospital Site, 610 University Ave., Toronto, Ontario, M5G 2M9, Canada Full list of author information is available at the end of the article
}

systems for the downregulation or overexpression of multiple targets have been adapted to address issues such as isoform redundancy, endogenous mutations of single shRNAs and successful targeting of signaling pathways [7-19].

Expression platforms have been constructed either using classical restriction-enzyme based cloning technology or novel recombination based technologies, of which the Gateway technology has gained an unprecedented use. Gateway utilizes elements of site-specific recombination of Escherichia coli bacteriophage lambda integrase/att to enable the transfer of genes between different vectors [20,21]. While many varieties of Gatewaycompatible vectors are available, problems may be encountered when either additional markers and/or different expression platforms are needed. This is especially the case for in vitro transformation experiments that are designed to introduce multiple genetic aberrations to cell lines in a stepwise fashion.

To enable efficient transfer of genes/shRNAs through different expression platforms, we have combined 
aspects of the Gateway system and restriction-based cloning technology and designed two entry vectors for either double shRNA or Cre/lox inducible gene expression and a series of lentiviral destination vectors containing an array of markers. We took advantage of already available and widely used expression/shRNA vectors to facilitate the conversion of reagents from one platform to the other. We have generated an additional, fully compatible system with currently available Gateway vectors which should further facilitate gene functional studies.

\section{Results and discussion}

Design of modified Gateway shRNA/gene expression system

We utilized a combination of restriction-based cloning and highly versatile Gateway site-directed recombination technology for the construction of modified
gene/shRNA expression system. This system consists of two main components: gene/shRNA entry and lentiviral destination vectors (Figure 1). Shuffling of inserts from entry vectors to any of lentiviral destination vectors is mediated by LR recombination reaction. Cloning details are provided in the Materials and Methods.

The double shRNA entry vector, pENTR.hU6.hH1, was designed by combining shRNA promoter cassettes from two published shRNA expression vectors, pSUPER. retro and plko.1.puro. One advantage of this strategy is the availability of shRNA cassettes based on the subcloning into pSUPER.retro or plko.1.puro. These cassettes are used for construction of the most extensively used shRNA libraries $[4,5]$. While these libraries have been applied successfully over the last 5 years, one of their major limitations is the extent of available selection markers. This is often the case in cell lines that require

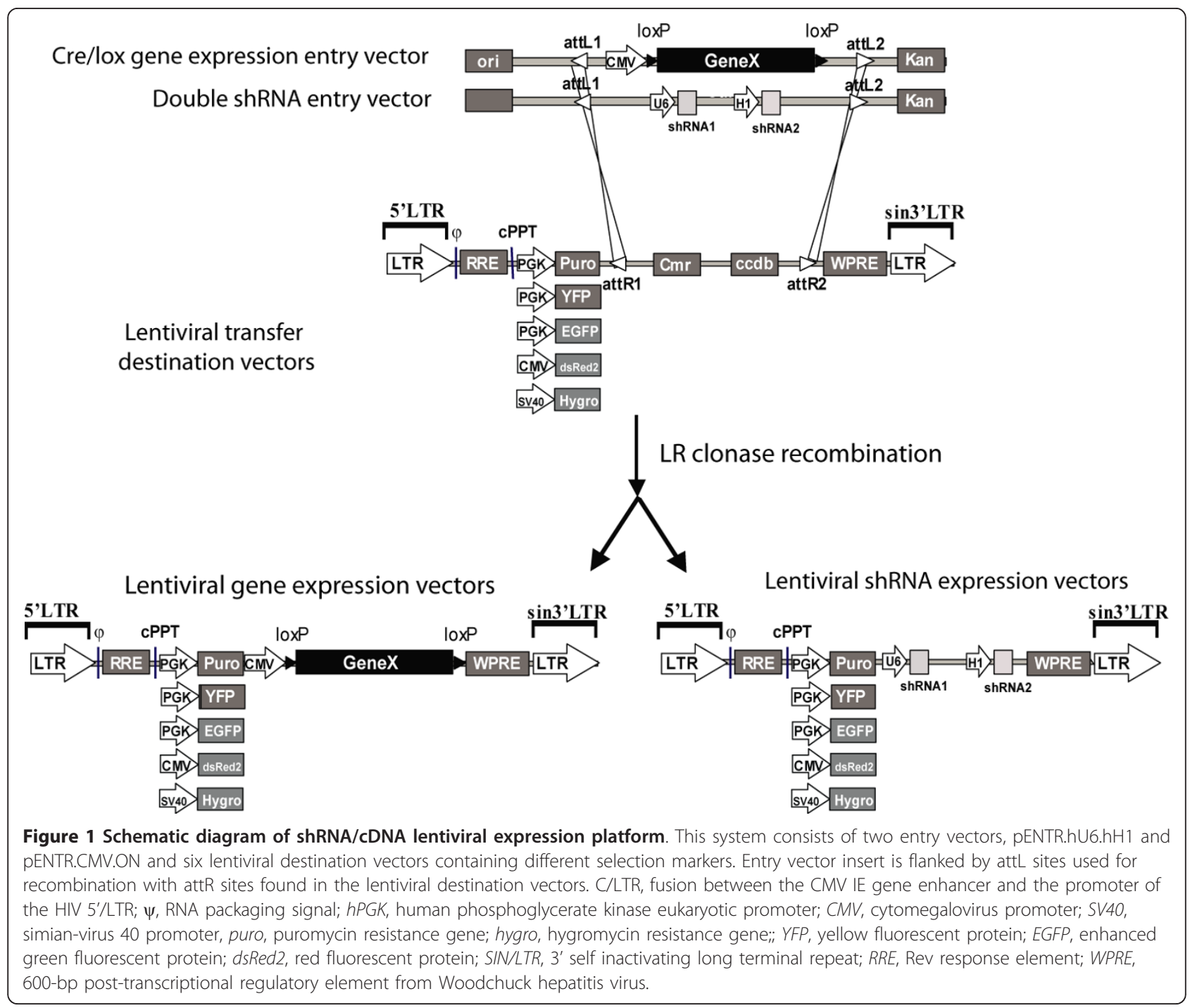


multiple shRNA or gene expression manipulations. Therefore, subcloning of shRNA libraries in a more versatile vector that allows for downstream transfer of shRNA cassettes to various expression platforms is highly desirable. Hence, the construction of pENTR. hU6.hH1 would allow for easy transfer of subcloned shRNA cassettes to different gateway based expression vectors, shRNA targeting of multiple genes, and the increased knockdown of a single target using multiple shRNA sequences. Furthermore, since pENTR.hU6.hH1 vector contains two different shRNA expression promoters, this would possibly prevent subsequent endogenous recombinations known to occur in shRNA vectors with the same tandem promoters [16].

Gene expression vector pENTR.CMV.ON is based on Cre/lox recombination and contains CMV promoter and MCS flanked by loxP sites. Most of the entry vectors available to date are promoterless and are recombined in promoter containing expression vectors. In addition, subcloning into these vectors can be accomplished by attB-PCR recombination avoiding restrictionbased cloning. While these vectors have been extensively used, they might not be adequate for certain applications. For example, attB-PCR requires very long primer sequences which sometimes results in labor intensive and time consuming PCR troubleshooting especially during amplifications of large DNA fragments. This might inadvertently increase a chance of possible transgene mutations arising during the PCR reaction. Hence, researchers would still have to resort to a more accurate restriction enzyme-based cloning. Furthermore, gene functional studies very frequently require the inducible transgene expression. To this end, we incorporated Cre/ LOX technology to our gene expression platform. The final entry vector, pENTR.CMV.ON (Figure 1), contains a potent CMV promoter which provides a better chance of gene overexpression as well as loxP sites required for Cre recombinase excision. To our knowledge, this is the first entry vector available for Cre/lox based gene expression.

The destination vectors constructed in this study (Figure 1) are based on the lentiviral gene expression which is the most utilized system to efficiently transduce hard to transfect cell lines or primary mammalian cells. Our destination vectors were engineered to express an array of selection markers through transgene independent promoters. While our system was under construction, two additional versatile Gateway based expression systems were reported [19,22]. Zhu et al. [19] constructed bicistronic lentiviral gateway destination vectors with internal-ribosome entry site (IRES) which coexpress the miR-shRNA with a variety of selection markers. While IRES has many advantages for the coexpression of transgenes, there have been some major limitations reported regarding their use. Several reports have shown that the IRES level of transgene expression depended considerably on the type of targeted cell $[23,24]$. Additionally, it was shown that IRES dependent translation could be affected by the first cistron with certain cistrons having inhibitory activity on IRES through yet undefined mechanisms [24,25]. Martin et al. [26] have also reported that several commercially available bicistronic vectors based on the IRES from Encephalomyocarditis virus (EMCV) have the $11^{\text {th }}$ AUG modified to a HindIII site to allow for easier subcloning [26]. However, this modification decreases dramatically the expression of the IRES-controlled coding sequence. Thus, our collection of lentiviral destination vectors will provide an alternative option to researchers faced with low level expression of the IRES-controlled transgene.

Campeau et al. have similarly developed an extensive array of lentiviral destination vectors [22] including promoterless pLentiX1 series which are compatible with our entry vectors. However, in this work we have constructed additional destination vectors expressing YFP, hygro and dsRed2 markers.

\section{Functionality of shRNA double promoter expression entry vector}

To demonstrate the efficacy of pENTR.hU6.hH1 construct we assessed its silencing effect on single and multiple gene targets. To test the functionality of both hU6 and $\mathrm{hH} 1$ promoters, we created three double promoter shRNA expression vectors with either shRNA targeting exon 2 of $\mathrm{p} 16^{\mathrm{INK} 4 \mathrm{a}}(\mathrm{p} 16 \mathrm{ex} 2)$ or nonsilencing shRNA (NS) under hU6 and/or hH1 (Figure 2A). These vectors were transiently transfected into 293T cells and the p16 mRNA expression levels were determined using reverse transcription-quantitative polymerase chain reaction $(\mathrm{Q}-$ RT-PCR). The mRNA knockdown was achieved when p16ex2 shRNA was expressed either under the hH1 or hU6 promoter when compared to control vector. Furthermore, there was a decrease in shRNA knockdown when using hH1 promoter, which is consistent with the previous reports of $\mathrm{hH} 1$ being a weaker promoter as compared to hU6 promoter [27]. Double expression of p16ex 2 significantly decreased the p16 mRNA levels in $293 \mathrm{~T}$ cells as compared to a single expression of p16ex2 under either promoter. The additive/double suppression of p16 mRNA could not be attributed to nonspecific effects of shRNA silencing since vectors coexpressing both p16ex 2 and control NS showed lower level of p16 knockdown as compared to vector doubly expressing p16ex2.

Our next experiment to test the functionality of pENTRhU6hH1 involved creating stable cell lines that would allow enough time for any possible shRNA recombinations known to arise in the targeted cells as a 


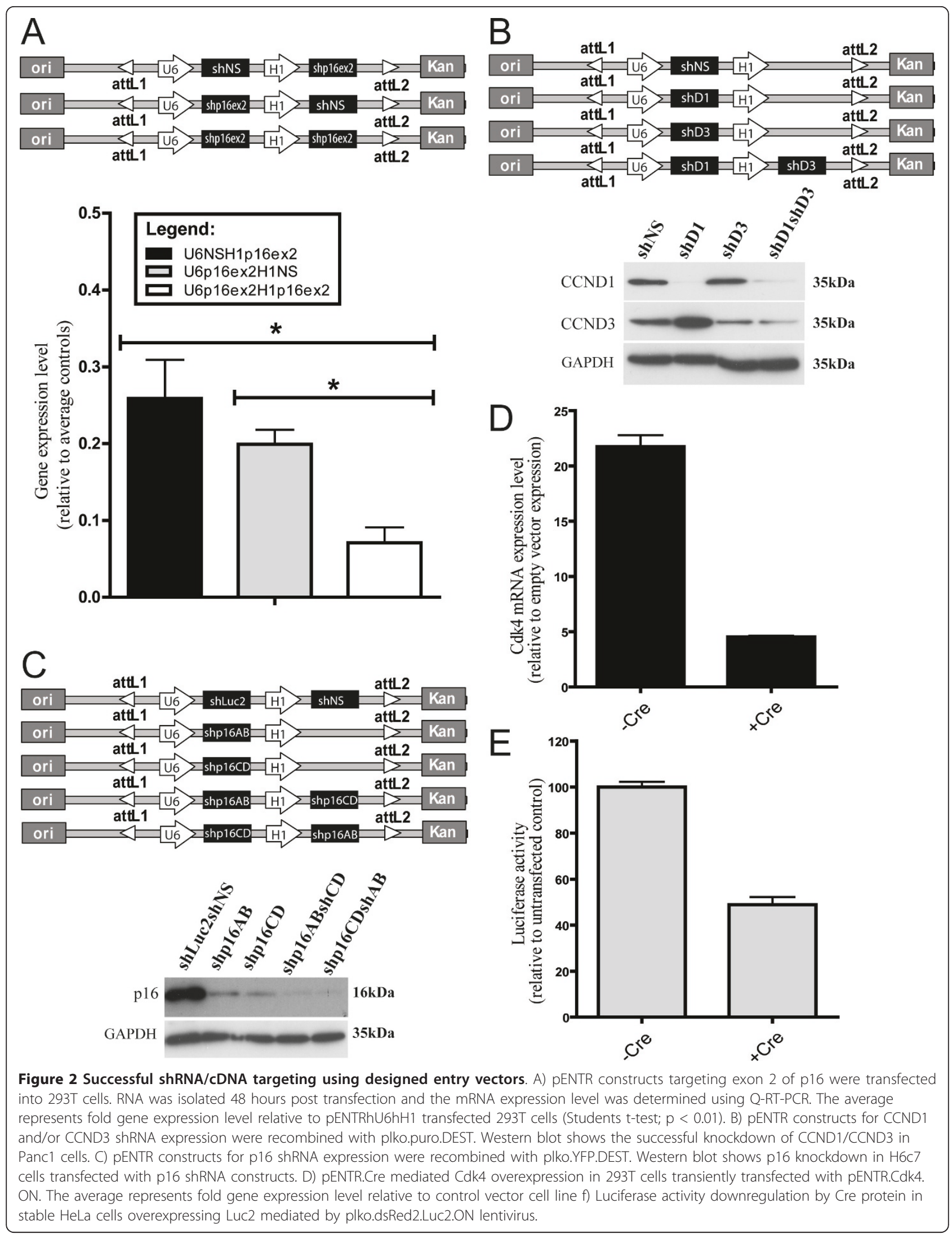


result of shRNA expression [16]. If such events occur using our system, then the knockdown efficiency would be greatly impaired. To test this, we chose shRNA against single targets, cyclin D1 (CCND1) and cyclin D3 (CCND3), which has been previously published [28]. We created three vectors pENTR.shD1, pENTR.shD3, pENTR.shD1.shD3 and control vector expressing nonsilencing shRNA, pENTR.shNS (Figure 2A). The resulting vectors were recombined with our destination vector pLKO.puro.DEST and lentiviruses were generated for transduction into the Panc-1 cell line. Western analysis on protein lysates from puromycin-selected Panc1 cells revealed specific knockdown of CCND1 and CCND3 with their respective shRNAs (Figure 2B). In addition, the Panc1 cell line transduced with shD1_D3 lentivirus showed significant knockdown of both cyclin D1 and cyclin D3 proteins with no significant loss of single shRNA effectiveness (Figure 2B). An upregulation of CCND3 in shD1 expressing Panc-1 cells was noted. We observed the same effects previously [28] and attributed it to a compensatory mechanism. These effects seem to be alleviated with the co-suppression of CCND3 as demonstrated in our current results (Figure 2B).

To determine whether our dual shRNA expression vector can be used to maximize the efficiency of gene silencing we targeted two different regions in exon 1 of $p 16^{\mathrm{INK} 4 \mathrm{~b}}$ mRNA. The experiments were performed on human pancreatic duct epithelial cell line $\mathrm{H} 6 \mathrm{c} 7$ that expresses very high levels of $\mathrm{p} 16^{I N K 4 a}$ due to loss of $\mathrm{Rb}$ secondary to HPV E6/E7 expression [29]. The efficient knockdown of $p 16^{\mathrm{INK} 4 \mathrm{a}}$ in H6c7 cells by shRNA has presented a major challenge. While we tested 16 different shRNAs against $p 16^{\mathrm{INK} 4 \mathrm{a}}$ (Additional file 1), none of the resulting H6c7 stable cell lines has shown greater than $60 \%$ reduction in $16^{I N K 4 a}$ levels. Additional technical difficulty in the specific silencing of $p 16^{\mathrm{INK} 4 \mathrm{a}}$ is that one can only target exon 1 , since exons 2 and 3 are shared with $p 14^{\mathrm{ARF}}$. This limits significantly the available mRNA sequence for siRNA design. To test if our dual shRNA expression vector would overcome these problems, four entry vectors expressing either single or combination of the most effective shRNAs against $p 16^{\text {INK4a }}$, shp16AB and shp16CD [30] were recombined with the plko.YFP.DEST vector (Figure 2C). H6c7 cells were transduced with the resulting lentiviruses and the p16 protein levels were assessed by western blotting. As predicted, the expression of two shRNAs against $p 16^{\mathrm{INK} 4 \mathrm{a}}$ led to a more effective suppression of the p16 ${ }^{I N K 4 a}$ level than a single shRNA cassette (Figure 2C). We also tested the actual p16AB and p16CD shRNA expression under different promoters using Q-RT-PCR (Additional File 2). While both promoters efficiently expressed $\mathrm{p} 16 \mathrm{AB}$ and $\mathrm{p} 16 \mathrm{CD}$ shRNA, there was a lower siRNA expression under $\mathrm{hH} 1$ promoter as compared to
hU6 promoter (Additional File 2). A number of factors should be considered prior to usage of the hH1 and hU6 promoters, the first being the potency of the shRNA used and the second being the cell type. The hH1 promoter should be employed for intermediate knockdown, while the hU6 can be used for more potent applications. Hence, our double shRNA expression vector can be particularly valuable for dose-dependent studies where variable expression is required.

\section{Functionality of Cre/lox inducible entry vector}

Next, we tested the functionality and efficiency of pENTR. CMV.ON vector using both transient and stable transfections. For transient expression testing, cyclin-dependent kinase 4 (Cdk4) was subcloned into pENTR.CMV.ON vector. The resulting pENTR.Cdk4.ON and empty control vector were transfected into $293 \mathrm{~T}$ cells. As measured by Q-RT-PCR, Cdk4 was overexpressed $(21.73 \pm 1.05$ fold $)$ compared to the empty vector control (Figure 2E). Additionally, co-transfection with the Cre expressing vector, pENTR.Cre, decreased the Cdk4 mRNA levels to $4.52 \pm$ 0.11 fold relative to the control vector (Figure 2E).

To create stable clones, we constructed vector expressing luciferase protein (pENTR.Luc2.ON) and recombined it with the plko.dsRed2.DEST vector, thus creating the destination clone plko.dsRed2.Luc2.ON. Lentiviruses generated using plko.dsRed2.Luc2.ON and control plko.dsRed2.DEST vectors were used to transduce HeLa cells, which were subsequently sorted for Red2 fluorescence. Luciferase protein was successfully overexpressed in HeLa-dsRed2.Luc2.ON cells as measured by Q-RT-PCR (data not shown) and luciferase assay (Figure 2F). Transient transfection of pENTR.Cre into these cells resulted in a significantly decreased luciferase activity (Figure 2F). Taken together these results demonstrated the functionality of both pENTR.CMV. ON and plko.dsRed2.DEST vectors.

\section{Functionality of lentiviral destination vectors}

While we successfully demonstrated the efficiency of destination vectors plko.puro.DEST, plko.YFP.DEST and plko.dsRed2.DEST (Figure 2), destination vectors expressing hygromycin (hygro) and green fluorescent protein (EGFP) remained to be tested.

To test the performance of remaining destination vectors, H6c7 stable cell lines expressing dsRed2 were generated using lentiviruses from plko.hygro.dsRed 2 and plko.EGFP.dsRed2 vectors. H6c7 cells were selected or sorted for their respective selectable markers and changes in red fluorescence were measured by flow cytometry. The plko.hygro.dsRed2 construct conferred resistance to hygromycin while plko.EGFP.dsRed2 expressed EGFP (data not shown). Furthermore, all H6c7 dsRed2 derived cell lines were positive for red 
fluorescence suggesting that dsRed 2 could be successfully overexpressed using any of our destination vectors (Figure 3A). The infection of $\mathrm{H} 6 \mathrm{c} 7 \mathrm{dsRed} 2$ derivatives using Adeno-Cre significantly reduced the expression of dsRed2 as measured by Red2 fluorescence (Figure 3A).

\section{Viral titer of destination vectors is dependent on the} insert size

It has been demonstrated that lentiviral titer depends greatly on the insert sequence length due to the packaging capacity of the lentivirus $[31,32]$. As a result multiple strategies have been employed over years to increase the viral titers of pseudotyped lentiviruses especially for gene therapy applications [33-36]. In order to fully characterize the efficacy of our expression system, we tested the viral titer of plko.YFP.DEST expressing genes of various sizes in 293T cells (Figure $3 \mathrm{~B})$. As predicted, our results showed that the viral titer is proportional to the insert size (Figure 3B). Although a destination vector with a large cDNA such as hTERT resulted ultimately in lower lentiviral titer, it did not impede the ability to efficiently overexpress the gene in stable H6c7 cell line (Figure 3C). Furthermore, it has been shown previously that low titer virus results in unprocessed and defective lentiviruses [35]. However, in our experiments, all YFP positive cells

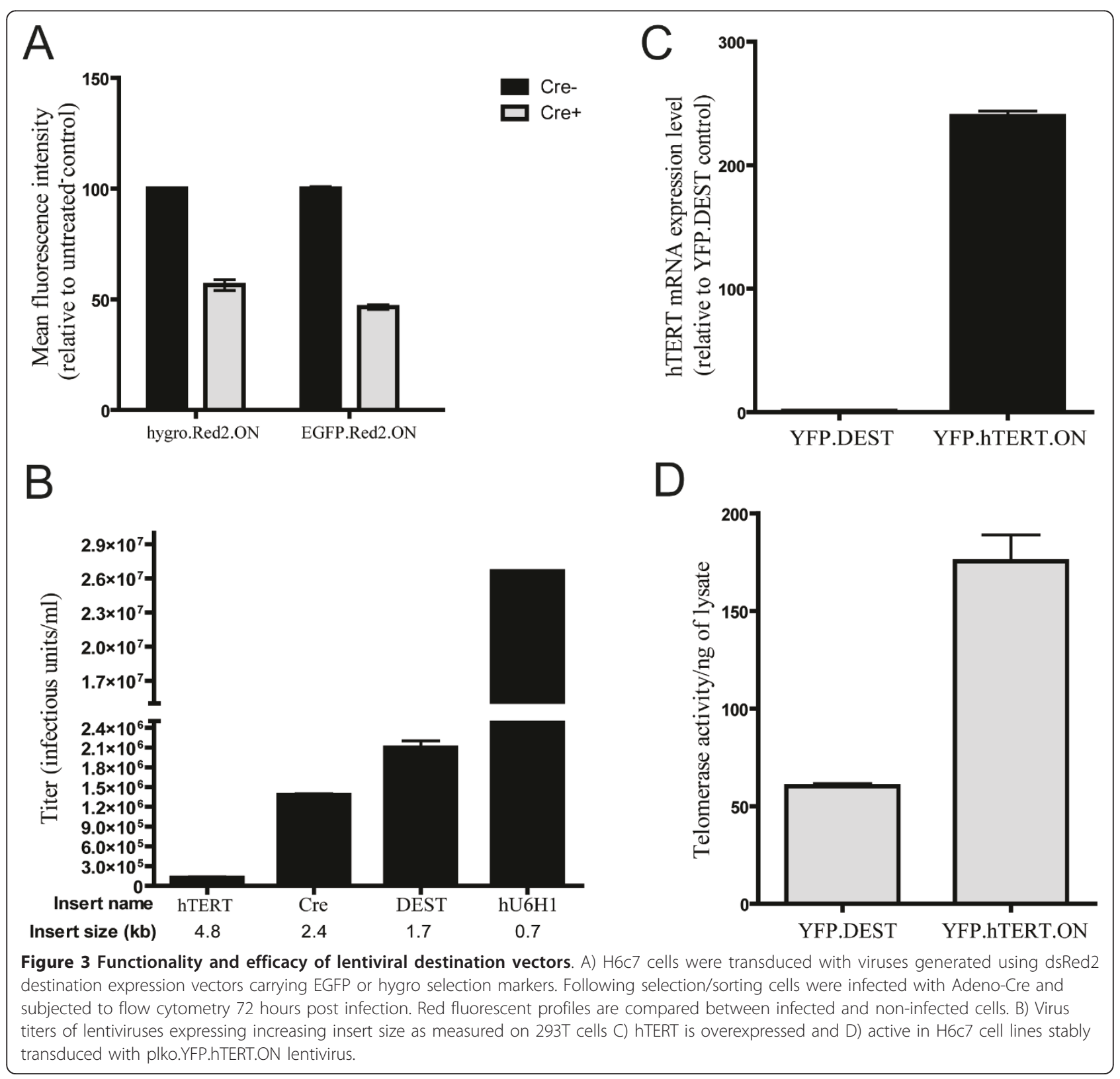


clearly overexpressed functional hTERT gene as determined by the hTERT activity assay (Figure 3D). Since the chance of vector integration event using non-viral expression system is generally very low, the use of low titer lentiviruses might still remain a better choice when creating stable cell lines.

\section{Conclusion}

Versatile gene/shRNA expression system is a necessity in gene functional studies. We have created a modified gateway lentiviral system for shRNA and Cre/lox inducible gene expression with entry and destination vectors that are compatible with existing gateway technologies. These reagents should provide additional choices for researchers studying many aspects of functional genomics.

\section{Methods \\ Vector construction}

All detailed maps and sequences of vectors constructed in this study are available upon request. Construction strategies, vector maps and restriction enzyme analysis were performed using Vector ${ }^{\text {NTI }}$ software (Invitrogen, Carlsbad, CA).

\section{Entry vectors construction}

The pENTR.hU6.hH1 was constructed in two steps. In the first step, XhoI digested hU6 cassette from plko.1puro [37] was ligated into Xhol/SalI linearized pENTR-4 vector (Invitrogen, Carlsbad, CA) to construct an intermediate single shRNA expression vector pENTR.hU6. Then, EcoRI/XhoI digested hH1 cassette from pSUPERretro [38] was inserted into EcoRI/XhoI linearized pENTR.hU6 vector creating double shRNA expression vector pENTR.hU6.hH1. We also constructed a second single shRNA expression vector pENTR-hH1 by removing hU6 cassette from pENTR.hU6.hH1 with NotI/ EcoRI. Blunt ends were then generated by Klenow reaction and subsequently ligated to make pENTR.hH1.

The pENTR.CMV.ON was constructed by inserting EcoRV/SnaBI linker cassette from pSicoR-puro-linkerloxp plasmid containing MCS flanked by loxP sites into HindIII/SnaBI linearized pENTR.CMV vector. HindIII site was blunt ended using Klenow reaction. Both intermediate plasmids pSicoR-puro-linker-loxp and pENTR. CMV vectors were constructed in our laboratory (data not shown).

\section{Destination vectors}

The plko.puro.DEST was constructed by inserting EcoRV/MluI digested attR1-cmr-ccdb-attR2 cassette from pTREX-DEST30 (Invitrogen) into ClaI/MluI digested plko.1-puro vector (ClaI blunt ended with Klenow enzyme). The plko.YFP.DEST was constructed by linearizing plko.YFP (gift from Dr. Linda Penn, Ontario Cancer Institute, Canada) with $A g e \mathrm{I} / E c o R I$ and ligating AgeI/NheI digestedattR1-cmr-ccdb-attR2 cassette from pTREX-DEST30. The EcoRI and AgeI sites were blunt ended using Klenow enzyme. The plko.dsRed2.DEST was made by inserting $S s p \mathrm{I} / N s i \mathrm{I}$ dsRed2 cassette from pSUPER.dsRed2 (constructed in our laboratory) into the $\mathrm{XbaI}$ (blunt ended)/NsiI digested plko.puro.DEST vector. The Destination vector plko.hygro.DEST was constructed in two steps. In the first step, an intermediate plasmid pBShygro was created by subcloning XmnI/SalI hygro cassette from SalI/EcoRV pIND/Hygro (Invitrogen) into pBluescriptIISK+ (Stratagene). Next, KpnI/SpeI hygro cassette from pBS-hygro was subcloned into plko.puro. DEST linearized with KpnI/SpeI creating plko.hygro. DEST vector. To construct plko.EGFP.DEST, the EGFP PCR fragment was (EGFP: F:5'TAGAATTCTACCGGGTAGGGGA3'; R: GGGGGTACCTCATTGGTCTTAAAGGTACCGA5') digested with the KpnI restriction enzyme at the 3' end and were then subcloned into the XcmI/KpnI linearized plko.puro.DEST plasmid.

\section{Subcloning into the entry vectors}

The pENTR.Luc.ON was constructed by inserting NheI/ XhoI luciferase fragment from pIND/HygroLuc (Clontech, Mountain View, CA) into AvrII/XhoI linearized pENTR-CMV-ON. The pENTR-dsRed2-ON was obtained by ligating dsRed2 fragment (HincII/XhoI) into HpaI/XhoI linearized pENTR-CMV-ON.

Oligos coding for the various shRNAs were annealed and cloned into either BglII/HindIII or AgeI/EcoRIdigested pENTRhU6hH1 as described previously [2]. All shRNAs used in this study are listed in Additional file 1.

\section{Transfer of $\mathrm{CDNA} / \mathrm{shRNA}$ cassettes to lentiviral destination vectors}

The constructs were designed to permit subcloning of shRNA/cDNA cassettes to different expression platforms by Gateway recombination. This involved direct LR recombination (Invitrogen, San Diego, CA) of the promoter containing shRNA/cDNA entry vector to any one of promortless lentiviral destination vectors with expression of different markers or drug selection genes.

\section{Bacterial strains and transformation}

For the construction of entry and destination vectors containing a toxic $c c d B$ gene competent Escherichia coli Library Efficiency DB3.1 ${ }^{\mathrm{TM}}$ cells (Invitrogen), were used for propagation according to the manufacturer's recommendations. For construction of other vectors and clones not containing the $c c d B$ gene, SUBCLONING EFFICIENCYTMDH5 $\alpha$ competent cells (Invitrogen) were used for transformation according to the supplier's instruction 


\section{Cell culture}

HeLa, Panc1 and 293T cells were obtained and cultured as recommended by the American Type Culture Collection (Rockville, MD). The Human pancreatic duct epithelial cell line (H6c7) was cultured as described previously [29].

\section{Transient transfections and stable cell line generations}

For transient transfection assays cells were transfected using Lipofectamine reagent (Invitrogen, Carlsbad, CA). For virus preparations, 293T cells were transfected using BD CalPhos Mammalian Transfection Kit (BD Biosciences). Both assays were performed as per the protocol provided by the manufacturer.

\section{Virus preparation, transduction and stable cell line generation}

Lentiviruses were prepared by transfecting three plasmids into 293T cells as described previously [39]. The plasmids are pMDLg/pRRE, the vesicular stomatitis virus (VSV-G) envelope plasmid pCMV-VSG, rev expressing plasmid pRSV-Rev and destination vectors containing the self-inactivating LTR. Stocks were stored frozen at $-80^{\circ} \mathrm{C}$ and tittered on $293 \mathrm{~T}$ cells.

Stable cell lines were isolated following viral transductions by either selection in the appropriate antibiotics for 1 - 2 weeks $(0.5 \mu \mathrm{g} / \mathrm{mL}$ puromycin; $50 \mu \mathrm{g} / \mathrm{mL}$ hygromycin; $100 \mu \mathrm{g} / \mathrm{mL}$ ) or by flow sorting using dual-laser FACSCalibur.

Adenovirus expressing Cre recombinant protein was purchased from Vector Biolabs (Philadelphia, PA) and used at 10 moi per cell for 72 hours.

\section{RNA isolation and Q-RT-PCR analysis}

RNA isolation and assay techniques used in this paper were published in our previous work [28]. Primers used in this study are listed in Additional file 1.

\section{Western analysis}

Immunoblotting was performed using whole protein extracts and probed with the following antibodies against cyclin D3 (BD Biosciences, San Jose, CA), cyclin D1 (BD Biosciences), p16 ${ }^{\mathrm{INK} 4 \mathrm{a}}$ (Cell Signalling, Boston, MA) and p14 ${ }^{\mathrm{ARF}}$ (NeoMarkers, Freemont, CA), GAPDH (Abcam, Cambridge, MA), and secondary mouse or rabbit conjugated IgG-horseradish peroxidase (Cell Signalling).

\section{Luciferase and hTERT assay}

Luciferase assay was performed using Luciferase Assay Kit (Promega Madison, WA). The hTERT activity was measured using TRAPEZE ${ }^{\circledR}$ RT Telomerase Detection Kit (Millipore, Billerica, MA). Both kits were used as recommended by the manufacters protocols.

\section{Flow cytometry}

The fluorescent emission intensities of EGFP, YFP and dsRed2 proteins were measured by FACS analysis using the FACSCalibur ${ }^{\mathrm{TM}}$ (BectonDickinson Corp.). Data were acquired and analyzed using CellQuest ${ }^{\mathrm{TM}_{\mathrm{V}}}$.3.0.

\section{Additional material}

Additional file 1: shRNA sequences and Q-RT-PCR primers used in this study.

Additional file 2: Figure S1: Comparison of shRNA expression under.

\section{Acknowledgements}

We thank Dr. Linda Penn for the initial gift of plko.YFP vector. Nikolina Radulovich is the Vanier Scholar of Canada (funded by Canadian Institutes of Health Research). Supported by the Canadian Institutes of Health Research grant MOP-49585 and partly by the Ontario Ministry of Health and Long Term Care. Dr. Tsao is the M. Qasim Choksi Chair in Lung Cancer Translational Research. The funding agencies have no direct input into the experimental design and performance as well as the drafting of this manuscript.

\section{Author details}

${ }^{1}$ University Health Network, Ontario Cancer Institute/Princess Margaret Hospital Site, 610 University Ave., Toronto, Ontario, M5G 2M9, Canada. ${ }^{2}$ Department of Laboratory Medicine and Pathobiology, Faculty of Medicine, University of Toronto, Medical Sciences Buildings, 1 King's College Circle, Toronto, Ontario, M5 S 1A8, Canada. ${ }^{3}$ Department of Medical Biophysics, University Health Network, Ontario Cancer Institute/Princess Margaret Hospital Site, 610 University Ave., Toronto, Ontario, M5G 2M9, Canada.

\section{Authors' contributions}

NR designed and constructed majority of the vectors, carried out the proofof-principle studies, and drafted the manuscript. LL constructed plko.EGFP. DEST and pENTR.Red2.ON vectors and assisted in drafting the manuscript. MT conceived and provided overall supervision of the study and edited the manuscript. All the authors have read and approved the final manuscript.

\section{Competing interests}

The authors declare that they have no competing interests.

Received: 2 September 2010 Accepted: 22 March 2011

Published: 22 March 2011

\section{References}

1. Elbashir SM, Harborth J, Lendeckel W, Yalcin A, Weber K, Tuschl T: Duplexes of 21-nucleotide RNAs mediate RNA interference in cultured mammalian cells. Nature 2001, 411(6836):494-498.

2. Brummelkamp TR, Bernards R, Agami R: A system for stable expression of short interfering RNAs in mammalian cells. Science 2002, 296(5567):550-553

3. Paddison PJ, Caudy AA, Bernstein E, Hannon GJ, Conklin DS: Short hairpin RNAs (shRNAs) induce sequence-specific silencing in mammalian cells. Genes Dev 2002, 16(8):948-958.

4. Bernards R, Brummelkamp TR, Beijersbergen RL: shRNA libraries and their use in cancer genetics. Nat Methods 2006, 3(9):701-706.

5. Root DE, Hacohen N, Hahn WC, Lander ES, Sabatini DM: Genome-scale loss-of-function screening with a lentiviral RNAi library. Nat Methods 2006, 3(9):715-719.

6. Moffat J, Grueneberg DA, Yang X, Kim SY, Kloepfer AM, Hinkle G, Piqani B, Eisenhaure TM, Luo B, Grenier JK, Carpenter AE, Foo SY, Stewart SA, Stockwell BR, Hacohen N, Hahn WC, Lander ES, Sabatini DM, Root DE: A lentiviral RNAi library for human and mouse genes applied to an arrayed viral high-content screen. Cell 2006, 124(6):1283-1298.

7. Freuler F, Stettler T, Meyerhofer M, Leder L, Mayr LM: Development of a novel Gateway-based vector system for efficient, multiparallel protein expression in Escherichia coli. Protein Expr Purif 2008, 59(2):232-241. 
8. Fux C, Langer D, Kelm JM, Weber W, Fussenegger M: New-generation multicistronic expression platform: pTRIDENT vectors containing sizeoptimized IRES elements enable homing endonuclease-based cistron swapping into lentiviral expression vectors. Biotechnol Bioeng 2004, 86(2):174-187.

9. Greber D, Fussenegger M: Multi-gene engineering: simultaneous expression and knockdown of six genes off a single platform. Biotechnol Bioeng 2007, 96(5):821-834.

10. Magnani E, Bartling L, Hake S: From Gateway to MultiSite Gateway in one recombination event. BMC Mol Biol 2006, 7:46.

11. Mitta B, Rimann M, Ehrengruber MU, Ehrbar M, Djonov V, Kelm J, Fussenegger M: Advanced modular self-inactivating lentiviral expression vectors for multigene interventions in mammalian cells and in vivo transduction. Nucleic Acids Res 2002, 30(21):e113.

12. Sasaki Y, Sone T, Yahata K, Kishine H, Hotta J, Chesnut JD, Honda T, Imamoto F: Multi-gene gateway clone design for expression of multiple heterologous genes in living cells: eukaryotic clones containing two and three ORF multi-gene cassettes expressed from a single promoter. J Biotechnol 2008, 136(34):103-112.

13. Scheich C, Kummel D, Soumailakakis D, Heinemann U, Bussow K: Vectors for co-expression of an unrestricted number of proteins. Nucleic Acids Res 2007, 35(6):e43.

14. Schubert S, Grunert HP, Zeichhardt H, Werk D, Erdmann VA, Kurreck J: Maintaining inhibition: siRNA double expression vectors against coxsackieviral RNAs. J Mol Biol 2005, 346(2):457-465.

15. Sone T, Yahata K, Sasaki Y, Hotta J, Kishine H, Chesnut JD, Imamoto F: Multi-gene gateway clone design for expression of multiple heterologous genes in living cells: modular construction of multiple cDNA expression elements using recombinant cloning. J Biotechno/ 2008, 136(34):113-121.

16. ter Brake O, 't Hooft K, Liu YP, Centlivre M, von Eije KJ, Berkhout B: Lentiviral vector design for multiple shRNA expression and durable HIV1 inhibition. Mol Ther 2008, 16(3):557-564

17. Wang S, Shi Z, Liu W, Jules J, Feng X: Development and validation of vectors containing multiple siRNA expression cassettes for maximizing the efficiency of gene silencing. BMC Biotechnol 2006, 6:50.

18. Yahata K, Kishine H, Sone T, Sasaki Y, Hotta J, Chesnut JD, Okabe M, Imamoto F: Multi-gene gateway clone design for expression of multiple heterologous genes in living cells: conditional gene expression at near physiological levels. J Biotechnol 2005, 118(2):123-134.

19. Zhu X, Santat LA, Chang MS, Liu J, Zavzavadjian JR, Wall EA, Kivork C, Simon MI, Fraser ID: A versatile approach to multiple gene RNA interference using microRNA-based short hairpin RNAs. BMC Mol Biol 2007, 8:98

20. Hartley JL, Temple GF, Brasch MA: DNA cloning using in vitro site-specific recombination. Genome Res 2000, 10(11):1788-1795.

21. Landy A: Dynamic, structural, and regulatory aspects of lambda sitespecific recombination. Annu Rev Biochem 1989, 58:913-949.

22. Campeau E, Ruhl VE, Rodier F, Smith CL, Rahmberg BL, Fuss JO, Campisi J, Yaswen P, Cooper PK, Kaufman PD: A versatile viral system for expression and depletion of proteins in mammalian cells. PLoS One 2009, 4(8):e6529.

23. Borman AM, Le Mercier P, Girard M, Kean KM: Comparison of picornaviral IRES-driven internal initiation of translation in cultured cells of different origins. Nucleic Acids Res 1997, 25(5):925-932.

24. Douin V, Bornes S, Creancier L, Rochaix P, Favre G, Prats AC, Couderc B: Use and comparison of different internal ribosomal entry sites (IRES) in tricistronic retroviral vectors. BMC Biotechnol 2004, 4:16.

25. Hennecke M, Kwissa M, Metzger K, Oumard A, Kroger A, Schirmbeck R, Reimann J, Hauser $\mathrm{H}$ : Composition and arrangement of genes define the strength of IRES-driven translation in bicistronic mRNAs. Nucleic Acids Res 2001, 29(16):3327-3334.

26. Martin P, Albagli O, Poggi MC, Boulukos KE, Pognonec P: Development of a new bicistronic retroviral vector with strong IRES activity. BMC Biotechnol 2006, 6:4.

27. Makinen $\mathrm{Pl}$, Koponen JK, Karkkainen AM, Malm TM, Pulkkinen $\mathrm{KH}$, Koistinaho J, Turunen MP, Yla-Herttuala S: Stable RNA interference: comparison of $\mathrm{U} 6$ and $\mathrm{H} 1$ promoters in endothelial cells and in mouse brain. J Gene Med 2006, 8(4):433-441.

28. Radulovich N, Pham NA, Strumpf D, Leung L, Xie W, Jurisica I, Tsao MS: Differential roles of cyclin D1 and D3 in pancreatic ductal adenocarcinoma. Mol Cancer 2010, 9:24.
29. Furukawa T, Duguid WP, Rosenberg L, Viallet J, Galloway DA, Tsao MS: Long-term culture and immortalization of epithelial cells from normal adult human pancreatic ducts transfected by the E6E7 gene of human papilloma virus 16. Am J Pathol 1996, 148(6):1763-1770.

30. Shin JJ, Katayama T, Michaud WA, Rocco JW: Short hairpin RNA system to inhibit human p16 in squamous cell carcinoma. Arch Otolaryngol Head Neck Surg 2004, 130(1):68-73.

31. Kumar M, Keller B, Makalou N, Sutton RE: Systematic determination of the packaging limit of lentiviral vectors. Hum Gene Ther 2001, 12(15):1893-1905.

32. Ni Y, Sun S, Oparaocha I, Humeau L, Davis B, Cohen R, Binder G, Chang YN, Slepushkin V, Dropulic B: Generation of a packaging cell line for prolonged large-scale production of high-titer HIV-1-based lentiviral vector. J Gene Med 2005, 7(6):818-834

33. Reiser J: Production and concentration of pseudotyped HIV-1-based gene transfer vectors. Gene Ther 2000, 7(11):910-913.

34. Sena-Esteves M, Tebbets JC, Steffens S, Crombleholme T, Flake AW: Optimized large-scale production of high titer lentivirus vector pseudotypes. J Virol Methods 2004, 122(2):131-139.

35. Kutner $\mathrm{RH}$, Zhang $X Y$, Reiser J: Production, concentration and titration of pseudotyped HIV-1-based lentiviral vectors. Nat Protoc 2009, 4(4):495-505.

36. Urbinati $F$, Arumugam $P$, Higashimoto $T$, Perumbeti A, Mitts K, Xia P,

Malik $P$ : Mechanism of reduction in titers from lentivirus vectors carrying large inserts in the 3'LTR. Mol Ther 2009, 17(9):1527-1536.

37. Stewart SA, Dykxhoorn DM, Palliser D, Mizuno H, Yu EY, An DS, Sabatini DM, Chen IS, Hahn WC, Sharp PA, Weinberg RA, Novina CD: Lentivirus-delivered stable gene silencing by RNAi in primary cells. RNA 2003, 9(4):493-501

38. Brummelkamp TR, Bernards R, Agami R: A system for stable expression of short interfering RNAs in mammalian cells. Science 2002, 296(5567):550-553

39. Dull T, Zufferey R, Kelly M, Mandel RJ, Nguyen M, Trono D, Naldini L: A third-generation lentivirus vector with a conditional packaging system. J Virol 1998, 72(11):8463-8471.

doi:10.1186/1472-6750-11-24

Cite this article as: Radulovich et al.: Modified gateway system for double shRNA expression and Cre/lox based gene expression. BMC Biotechnology 2011 11:24.

\section{Submit your next manuscript to BioMed Central and take full advantage of:}

- Convenient online submission

- Thorough peer review

- No space constraints or color figure charges

- Immediate publication on acceptance

- Inclusion in PubMed, CAS, Scopus and Google Scholar

- Research which is freely available for redistribution

Submit your manuscript at www.biomedcentral.com/submit
C Biomed Central 